\title{
El acuerdo MERCOSUR-Unión Europea: un abordaje multicausal de la conclusión del proceso negociador
}

\author{
María Victoria ÁlVAREZ \\ Y JULIETA ZELICOVICH*
}

\begin{abstract}
RESUMEN
El objetivo de este trabajo es dilucidar cuáles fueron los factores que permiten explicar la conclusión del acuerdo entre MERCOSUR y la Unión Europea, en junio de 2019, tras 20 años de negociaciones. De igual manera, propone indagar en si el predominio de alguno de dichos factores ha generado efectos sobre los usos estratégicos que las partes hacen del acuerdo negociado y su (posible) ratificación. Se postula una explicación tripartita y multicausal conforme a la cual el acuerdo MERCOSUR-Unión Europea fue posibilitado por tres tipos de factores: los intereses materiales económicos y comerciales, la interacción estratégica y geopolítica, y la identidad birregional. Esta convergencia de factores implica que el trade-off que permitió llegar al acuerdo es multivariado pero no implica que sea necesariamente armónico. Así, se sugiere que según sea el elemento privilegiado por las partes involucradas, el acuerdo adquiere distintos significados y diferentes perspectivas de ratificación. El acuerdo ha sido presentado como un instrumento para el acceso a los mercados; como una oportunidad para incrementar el bienestar y eficiencia de los distintos sectores económicos. Desde lo estratégico y geopolítico, como un hito para la conservación del orden liberal internacional. En tanto que el elemento identitario permite sostener que el acuerdo entre MERCOSUR y la Unión Europea contribuye a la consolidación de una identidad común en torno a un conjunto de valores compartidos. El trabajo presenta un diseño metodológico cualitativo, basando en el análisis documental. El debate presentado se inscribe en la discusión de los determinantes del regionalismo y la cooperación en materia de integración regional dentro de la disciplina de las Relaciones Internacionales. El trabajo pone de manifiesto que, en un contexto donde las negociaciones comerciales internacionales son cada vez más complejas y donde la variable comercial ha dejado de ser un tema de low agenda, un análisis multicausal con marcos conceptuales eclécticos presenta ventajas. Con este abordaje, la investigación realizada permite echar luz sobre las tensiones subyacentes al momento de la firma del acuerdo, y que comienzan a manifestarse en el proceso de ratificación. Muestra también que sobre un mismo hecho de la política internacional-el acuerdo comercial- coexisten distintas miradas y motivaciones por parte de los actores involucrados. El artículo se organiza de la siguiente manera: luego de la introducción presentamos las definiciones relativas a nuestro marco teórico y encuadre metodológico. En el primer apartado nos centramos en la hipótesis concerniente a los intereses materiales económicos y comerciales. El segundo apartado, toma, por su parte, la de la variable geopolítica y estratégica. La tercera sección expone las características e implicancias del vínculo interregional. Finalmente, se presentan las conclusiones de la investigación.
\end{abstract}

\section{Palabras clave}

Regionalismo; negociaciones comerciales; MERCOSUR; Unión Europea; interregionalismo.

\section{TITLE}

The MERCOSUR-European Union agreement: a multi-causal approach to the conclusion of the negotiating process

\section{Abstract}

On 28 June 2019, the Southern Common Market (MERCOSUR) and the European Union (EU) concluded the negotiations on the trade chapter of the Association Agreement that both blocs had been working on for twenty years. The conclusion of the negotiations, announced at the G20 summit in Osaka, surprised everyone. They had begun in 1999 but were interrupted (and subsequently retaken) on several occasions due to differences in key issues such as agriculture, services and government procurement. Although a trade agreement was

DOI:

http://doi.org// 0.15366/relacionesinternacionales2020.44.006

Formato de citación recomendado:

ÁLVAREZ, María Victoria y ZELICOVICH, Julieta (2020), "El acuerdo MERCOSUR-Unión Europea: un abordaje multicausal de la conclusión del proceso negociador", Relaciones Internacionales, $n^{\circ} 44$, pp. I07-I 25.
* María Victoria ÁLVAREZ, Doctora en Relaciones Internacionales, Universidad Nacional de Rosario (Argentina). Directora del Grupo de Estudio sobre la Unión Europea, Instituto de Investigaciones, Facultad de Ciencia Política y Relaciones Internacionales (UNR). Coordinadora del Proyecto Jean Monnet "Acercando la Unión Europea a los Estudiantes y la Sociedad Civil”.

Julieta ZELICOVICH, Doctora en Relaciones Internacionales, Universidad Nacional de Rosario (Argentina). Investigadora del Consejo Nacional de Investigaciones Científicas y Técnicas (CONICET), Argentina. Directora del Grupo de Estudio sobre Negociaciones Comerciales Internacionales, Instituto de Investigaciones, Facultad de Ciencia Política y Relaciones Internacionales (UNR).

Recibido:

23/12/2019

Aceptado: I4/05/2020 
reached in June 2019, we should note that this does not mean the end of the process, as ratification by the parties and entry into force is still pending.

At the time of the conclusion of the "agreement in principle", the EU faced the consequences of a multiplicity of overlapping crises and concentrated its foreign policy mainly on its eastern and southern neighbors, while the South American bloc had diverted its external relations to Asia.When everything suggested that MERCOSUR and Europe were drifting apart, they managed to reach the agreement.What were the determining factors for this outcome?

The explanation of why countries decide to close a trade agreement leads to a diversity of factors. In the case of the agreement between MERCOSUR and the EU, it was exhibited as an instrument for market access, and as an opportunity to increase the well-being and efficiency of different economic sectors. From the strategic and geopolitical point of view, the agreement was presented as a milestone for the preservation of the international liberal order.With regard to the identity element, the agreement contributes to the consolidation of a bi-regional common identity around a set of shared values.

The MERCOSUR-EU agreement, then, does not allow a single appraisal, but it can be elucidated by the presence of at least three kinds of explanatory factors: economic, strategic and geopolitical, and also ideational. A large and growing body of literature on international cooperation, economic integration and inter-regionalism has investigated the selected factors.

Liberal intergovernmentalists and international political economy scholars have studied the interaction between economic and geopolitical interests. In the economic integration process, according to these approaches, economic interests take precedence. However, they recognize that in cases where economic interests are indeterminate or diffuse, other objectives might be relevant (such as geopolitical, strategic and, we will add, ideational). In this study, we analyze how these three factors have been present to account for the agreement reached and what their implications are.

The aim of this research, then, is to investigate the factors that explain the conclusion of the agreement between MERCOSUR and the European Union, in June 2019, after twenty years of negotiations. Similarly, it attempts to examine whether any of these factors have an impact on the strategic uses that the parties make of the negotiated agreement and its (possible) ratification.

Our hypothesis is that the conclusion of a deal between MERCOSUR and the EU is a multi-causal process involving simultaneously three types of factors: economic and trade material interests, strategic and geopolitical interaction, and bi-regional identity. The convergence of factors implies that the trade-off that allowed the agreement to be reached is multivariate, which does not suggest that it is necessarily harmonious. Each of these factors contributes to giving a different meaning or perspective, making available different strategic uses of the agreement by the countries involved.These different uses and meanings attributed to the negotiated agreement on the basis of the factors involved may also affect the dynamics of the ratification process.

In methodological terms, the article follows the logic of a qualitative case study. The study is longitudinal, seeking to understand the conclusion of the negotiations while still considering the phenomenon over time. The techniques of data collection and analysis were qualitative, relying on the review of official documents, statements and speeches of the various actors involved, as well as on secondary statistical and bibliographic sources.

The study shows that multi-causal analyses with eclectic conceptual frameworks have advantages, in a context where international trade negotiations are becoming more complex and where trade has ceased to be a low agenda issue. Therefore, the research carried out makes it possible to shed light on the tensions underlying the signing of the agreement, which also appear in the ratification process. Moreover it shows that different actors' viewpoints and motivations coexist regarding the same international policy event -the trade agreement-

This study provides support for our initial argument. On the economic and commercial dimensions, the parties involved managed to bring their positions closer on the most sensitive issues, which - until then- appeared as insurmountable differences. While the structural characteristics of trade and investment did not change and nor did the trade preferences of the sectors, there was a change in the political dimension of these elements. From this perspective, the ratification of the agreement is imperative, but it is precisely because of the sensitivities involved that a higher level of politicization is expected.

Geopolitics has also played a relevant role, especially in the strategic use of the announcement of the agreement at the Osaka Summit.Thus, it was presented as a milestone for the preservation of the international liberal order, in contrast to the dynamics of the Sino-American trade war.This strategic game, however, is satisfied with the mere announcement of the arrangement and the geopolitical variable does not generate the same incentives for ratification as it did for the conclusion of the negotiations.

Finally, the explanation based on the identity element is constructed from inter-regionalism and makes it possible to argue that the agreement between MERCOSUR and the European Union represents a milestone in the consolidation of a common identity around a set of shared values. However, these are a precondition for the agreement to take place, and do not fully explain the 2019 outcome.

Putting these three variables into dialogue has proved indispensable to apprehend the complexity of a long-standing process, and the implications of an eventual ratification.These findings, however, raise new questions. Future work could enrich the study of the MERCOSUREU agreement, including the challenges of politicization.Also, since the study suggests a link between the three explanatory factors and the uses of the trade agreement, in future investigations it might be possible to undertake comparative studies between different processes of international trade negotiations.

The article has been organised in the following way. After the introduction, the paper goes on to present the definitions related to the conceptual framework and research design. In the first section we focus on the hypothesis regarding material economic and commercial interests. The second section evaluates the geopolitical and strategic variable.The third section presents the characteristics and implications of the interregional link. Finally, we present the conclusions of the investigation.

\section{KEYWORDS}

Regionalism; trade negotiations; MERCOSUR; European Union; interregionalism. 
I

\section{ntroducción}

La conclusión de las negociaciones comerciales entre el Mercado Común del Sur (MERCOSUR) y la Unión Europea (UE), anunciada en la cumbre del G-20 en Osaka (Japón) a fines de junio de 2019 , sorprendió a propios y ajenos. Las negociaciones habían comenzado en 1999 pero se estancaron en octubre de 2004 debido a las diferencias en cuestiones claves como el comercio en la agricultura, los servicios y la apertura de los mercados de contratación pública. Las conversaciones se reanudaron con poco éxito en 2010 en la Cumbre euro-latinoamericana de Madrid, y nuevamente fueron relanzadas en 2016. Finalmente, concluyeron con la firma de un acuerdo en principio el 28 de junio de 2019.

Para ese entonces, la UE afrontaba desde hacía una década una multiplicidad de crisis superpuestas (Álvarez, 20I3) y concentraba su política exterior principalmente en sus vecinos del este y el sur, mientras que el bloque sudamericano había desviado sus relaciones externas hacia Asia-Pacífico (Ortiz Velásquez y Dussel Peters, 2016). En el momento en que parecía que, ante la falta de impulso e ideas, el MERCOSUR y Europa se estaban distanciando, lograron alcanzar el acuerdo. ¿Cuáles fueron los factores determinantes de este resultado?

El objetivo de este trabajo es dilucidar cuáles son los factores que permiten explicar la conclusión del acuerdo entre los dos bloques regionales. Asimismo, indagar si el predominio de alguno de dichos factores genera efectos sobre los usos estratégicos que las partes hacen del acuerdo negociado y su (posible) ratificación. Con ello, el trabajo se inscribe en el debate relativo a los determinantes del regionalismo, el interregionalismo y la cooperación en materia de integración regional de larga trayectoria en la disciplina de las Relaciones Internacionales'.

Nuestro argumento es que la finalización de las negociaciones por el acuerdo MERCOSURUE es un proceso multicausal en el que intervienen de forma simultánea tres factores: los intereses materiales económicos y comerciales, la interacción estratégica y geopolítica, y la identidad birregional. Cada uno de ellos contribuye a narrar un relato acerca del acuerdo, dando lugar a distintos usos estratégicos del mismo por parte de los países que lo integran. Estos distintos usos y significados atribuidos al acuerdo negociado en razón de los factores intervinientes pueden incidir, además, en las dinámicas del proceso de ratificación ${ }^{2}$.

En definitiva, se trata de una hipótesis tripartita y multicausal. No nos proponemos testear si una teoría es más importante que otra; tampoco afirmar que se tratan de explicaciones excluyentes. Consiste más bien en un esfuerzo por "relevar la importancia relativa de los distintos factores intervinientes" (Moravcsik, 1998, p.12), como lo sugiere Moravcsik, en The Choice for Europe (1998).

Esta decisión de corte teórico y metodológico responde a la particularidad del caso en cuestión. Habiéndose iniciado las negociaciones en la década del noventa, el acuerdo MERCOSURUE ha sido considerado parte de las negociaciones de los acuerdos del "nuevo regionalismo", ola

\footnotetext{
La literatura sobre el acuerdo MERCOSUR-UE es vasta, demostrando el interés de la academia en el seguimiento y problematización de las negociaciones en todas sus etapas. En este trabajo nos centraremos en la coyuntura reciente, dialogando a lo largo del manuscrito con las investigaciones que discuten el cierre del acuerdo y sus implicancias.

2 Entendida como la posibilidad de mayor o menor complejidad en los tiempos y en la politización de la ratificación, por oposición a un modelo determinístico —ratificación o no ratificación-.
} 
dentro de la cual el factor predominante ha sido el económico ${ }^{3}$. No obstante, ninguna de las negociaciones iniciadas en dicha ola perduró por veinte años, ni tuvo entre las partes negociadoras el rasgo interregional ${ }^{4}$. Además, de entre todas las subregiones latinoamericanas, el MERCOSUR es el que tiene los lazos históricos y culturales más sólidos y profundos con Europa.

De estas particularidades en el vínculo, y del momento singular de la firma, es que creemos necesario avanzar hacia explicaciones multicausales del resultado acaecido en junio de 20195 . Este acuerdo en principio constituye una pieza central de la estrategia de vinculación que ambas regiones comenzaron a planear en los años noventa y que desde entonces, con sus idas y vueltas, ha marcado la cadencia de la relación.

Los tres factores seleccionados reconocen una trayectoria arraigada en las investigaciones sobre cooperación internacional, interregionalismo e integración económica. La interacción entre intereses económicos y geopolíticos ha sido estudiada en la literatura tanto por el intergubernamentalismo liberal, como por la economía política internacional, donde destacan los trabajos de Helen Milner (1999), Mansfield y Milner (1999), Robert Gilpin (200I), Gruber (200I), Laïdi (2008) o Dieter (2014) entre otros. En los procesos de integración económica comercial, según estos enfoques, los intereses económicos tienen primacía. No obstante, reconocen que en casos en los que los intereses económicos son indeterminados o difusos, se puede dar lugar a otro tipo de objetivos (como los geopolíticos, estratégicos y -agregamos - identitarios). Nos proponemos en este estudio analizar de qué forma han estado presentes estos tres factores y cuáles son sus implicancias.

En cuanto a los intereses materiales, éstos corresponden a una configuración doméstica de actores que pujan por la política exterior. Responden a las movilizaciones de los sectores privados y elites políticas, fundamentalmente. Tienden a generar una presión de abajo hacia arriba. Para teorías como el intergubernamentalismo liberal $-y$ también para algunas corrientes del neofuncionalismo-, los intereses económicos y materiales son una condición necesaria para que la integración funcione (Mattli, 1999; Laursen, 2008; Malamud, 2018).

Los factores geopolíticos y estratégicos son, por oposición a los anteriores, externos a los estados parte, y posiblemente más volátiles. No son movilizados por demandas de sectores privados o de la sociedad civil sino que son proveídos por los ejecutivos de los gobiernos que llevan adelante la negociación, conforme los condicionantes sistémicos y las percepciones que de éstos tienen esas élites. Se trata de concebir a los acuerdos de comercio como una acción estratégica con fines políticos. En la literatura, la existencia de liderazgos hegemónicos, la presencia de instituciones, los valores y principios compartidos, la permanencia (o remoción) del status quo, e incluso cuestiones de seguridad, han sido señalados como factores que pueden explicar la propensión de los estados a establecer negociaciones comerciales internacionales y firmar acuerdos entre sí.

3 Sobre esta ola de acuerdos comerciales véase OMC (20II).

4 De hecho, las negociaciones entre UE y Comunidad Andina, o entre UE y ASEAN no lograron avanzar sobre ese formato, a pesar de ser el interregionalismo un componente clave en la aproximación inicial (Meissnier, 20I5).

5 En la línea de explicaciones multicausales en materia de acuerdos comerciales puede destacarse el trabajo de Gómez-Mera (2009); también Moravcsik (1998) mencionado supra. 
La búsqueda de acuerdos comerciales como reacción estratégica a una crisis de hegemonía fue una interpretación bastante extendida en la década de los noventa (Mansfield y Milner, 1999). De cara al siglo XXI, también se ha vinculado la celebración de las negociaciones comerciales internacionales con las disputas de poder en torno a la gobernanza global del comercio internacional (Bouzas y Zelicovich, 20I4). El acuerdo entre el MERCOSUR y la UE sería también la expresión de una nueva lectura por parte de estos actores respecto de la distribución del poder mundial y del rol de los regionalismos en la gobernanza global.

En tercer lugar se considera al interregionalismo y a la identidad como variables explicativas del acuerdo negociado (Farrell, Hettne y Van Langenhove, 2005; Söderbaum y Van Langenhove, 2005; Hänggi, Roloff y Rüland, 2006; Hettne, 20I4; Rüland, 20I4). Al analizar las mismas distinguiremos tres cuestiones: la cuestión identitaria en el MERCOSUR en su dimensión externa, la proyección externa de la identidad de la UE y las consecuencias para su estrategia regionalista e interregionalista $y$, finalmente, la posibilidad de crear identidades compartidas entre dos actores a través del interregionalismo (Hänggi, Roloff y Rüland, 2006). El interregionalismo alude al establecimiento de estrechos vínculos institucionales entre dos bloques regionales. Se trata de un proceso relativamente nuevo en el escenario internacional, siendo a partir de los años noventa -con un escenario de post Guerra Fría y de globalización-cuando se visualiza con mayor intensidad.

Los actores involucrados en vínculos interregionales pueden perseguir objetivos variados. En particular, la perspectiva constructivista considera la construcción de estructuras normativas e identidades como componente crucial de estas relaciones (Söderbaum y Van Langenhove, 2005). El interregionalismo puede alentar dinámicas de fortalecimiento de la cohesión y la identidad del grupo. La cohesión del actor regional impacta en la identidad y la "conciencia" regional a través de las expectativas y reacciones de terceros (Hettne, 20I4, p. 57). La interacción puede resaltar las diferencias entre "nosotros" y "ellos", sea el otro grupo, o un tercero al que ambos perciben como amenaza, y estimular la cohesión interna a partir de valores y normas compartidas (Sanahuja, 2007, p. 8).

De acuerdo con la hipótesis enunciada, en el caso de las negociaciones MERCOSUR-UE consideramos que ninguno de estos tres elementos que las distintas teorías presentan como posibles razones para impulsar un acuerdo comercial son, por sí solos, suficientes para explicar el acuerdo. Desde una perspectiva de multicausalidad, argumentamos que los tres están presentes y son necesarios de manera conjunta para entender la firma del acuerdo de junio de 2019 .

Por su parte, esta convergencia de factores implica que el trade-off que permitió llegar al acuerdo es multivariado pero no implica que sea necesariamente armónico. Según cuál sea el elemento privilegiado por las partes involucradas, el acuerdo adquiere distintos significados y diferentes perspectivas de incentivos para el proceso de ratificación. Desde la visión que privilegia a los intereses materiales económicos y comerciales, las negociaciones se concluyeron como instrumento de apertura de mercados y de integración comercial entre ambos bloques. A partir del foco en los factores estratégicos y geopolíticos, el acuerdo entre el MERCOSUR y la Unión Europea es una herramienta política:un mensaje o posicionamiento en la disputa por la gobernanza global, en el contexto de la crisis de la globalización. Para el tercer enfoque, que alude a elementos 
identitarios, el acuerdo, tras veinte años de negociaciones, se concluye como mecanismo para la consolidación de una relación interregional basada en valores e identidades comunes.

El desarrollo de estos argumentos aplicados a las negociaciones MERCOSUR-UE sigue la lógica de un estudio de caso de carácter cualitativo. El recorte temporal es de tipo longitudinal, buscando comprender la conclusión de las negociaciones sin dejar de considerar el fenómeno a lo largo de su desarrollo en el tiempo. Las técnicas de recolección y análisis de datos fueron de tipo cualitativas, apoyándose en la revisión de documentos, declaraciones y discursos de los distintos actores involucrados, así como en fuentes secundarias estadísticas y bibliográficas.

Tras estas consideraciones teóricas y metodológicas, en el siguiente apartado nos centramos en la hipótesis concerniente a los intereses materiales económicos y comerciales. El segundo apartado, toma, por su parte, la de la variable geopolítica y estratégica. La tercera sección expone las características e implicancias del relato que valora el vínculo interregional. Finalmente, se presentan las conclusiones de la investigación.

\section{MERCOSUR-UE como instrumento económico comercial}

Las negociaciones entre el MERCOSUR y el bloque europeo, lanzadas en 1999, estuvieron guiadas por el Acuerdo Marco de Cooperación de 1995. Este acuerdo planteaba una vinculación auténticamente de "bloque a bloque", siendo así el primer acuerdo entre dos uniones aduaneras dotadas de subjetividad jurídica internacional. La meta era establecer un acuerdo de libre comercio de tipo OMC plus, es decir, un acuerdo que profundizara el alcance de los compromisos negociados en el marco de la Ronda Uruguay unos años antes. Se trataba de un instrumento que apuntase a la:

"liberalización [...] del comercio de bienes y servicios, [...] mejorar el acceso a compras gubernamentales [...], un ambiente no discriminatorio en las inversiones, [...] adecuada y efectiva protección a los derechos de propiedad intelectual, [...] políticas de competencia [...] y adecuadas y efectivas disciplinas en el cambio de los instrumentos de defensa comercial" (Comité de Negociaciones Birregionales MERCOSUR-Unión Europea, 2000).

Adicionalmente se establecía la inclusión de un mecanismo de solución de controversias, y el seguimiento de tres principios generales a lo largo del proceso negociador:"negociaciones abarcativas y resultados equilibrados; la no exclusión de ningún sector, teniendo en cuenta las sensibilidades de ciertos productos y servicios de conformidad con las reglas de la OMC; y la noción de compromiso único" (Comité de Negociaciones Birregionales MERCOSUR-Unión Europea, 2000).

Detrás de este proceso se articulaban distintos intereses materiales -económicos y comerciales-, que daban impulso a la negociación o generaban resistencias a la misma, según cómo se ubicasen las preferencias. Destacamos tres características distintivas de los intereses materiales de las negociaciones entre MERCOSUR y la Unión Europea, que han marcado esas oscilaciones: la asimetría relativa entre las partes involucradas; la existencia de complementariedad entre ambas economías; y la divergencia en las preferencias políticas detrás de esa complementariedad.

La asimetría ha sido central en la disputa por el trato especial y diferenciado y en el 
margen de maniobra de cada uno en el proceso de negociaciones. Los intercambios entre Europa y MERCOSUR se han caracterizado por la diferencia de los volúmenes relativos de los flujos y por el contenido de tipo inter-industrial de las canastas exportadoras 6 . Mientras que MERCOSUR es tan solo el $2 \%$ de las exportaciones de la UE, para el bloque sudamericano las exportaciones hacia el viejo continente representan el $16 \%$ del total de ventas ${ }^{7}$ (Gráfico I). Del lado de las importaciones, los productos de la UE representan el $21 \%$ de las importaciones del MERCOSUR del año 20I7, en tanto que las ventas del MERCOSUR a Europa son el $2 \%$ del total importado por esta última. El patrón de los intercambios se concentra en productos primarios y de bajo valor agregado por parte del MERCOSUR, tales como porotos y pellets de soja, pasta química de madera, minerales de hierro, aceite de petróleo, jugo de naranja y carne bovina enfriada. Los principales productos exportados por la UE a MERCOSUR son, en cambio, medicamentos, aeronaves, autopartes, productos farmacéuticos, embarcaciones, preparaciones de petróleo, fungicidas (LSE Consulting, 2018).

\section{Gráfico I: Exportaciones MERCOSUR-UE}

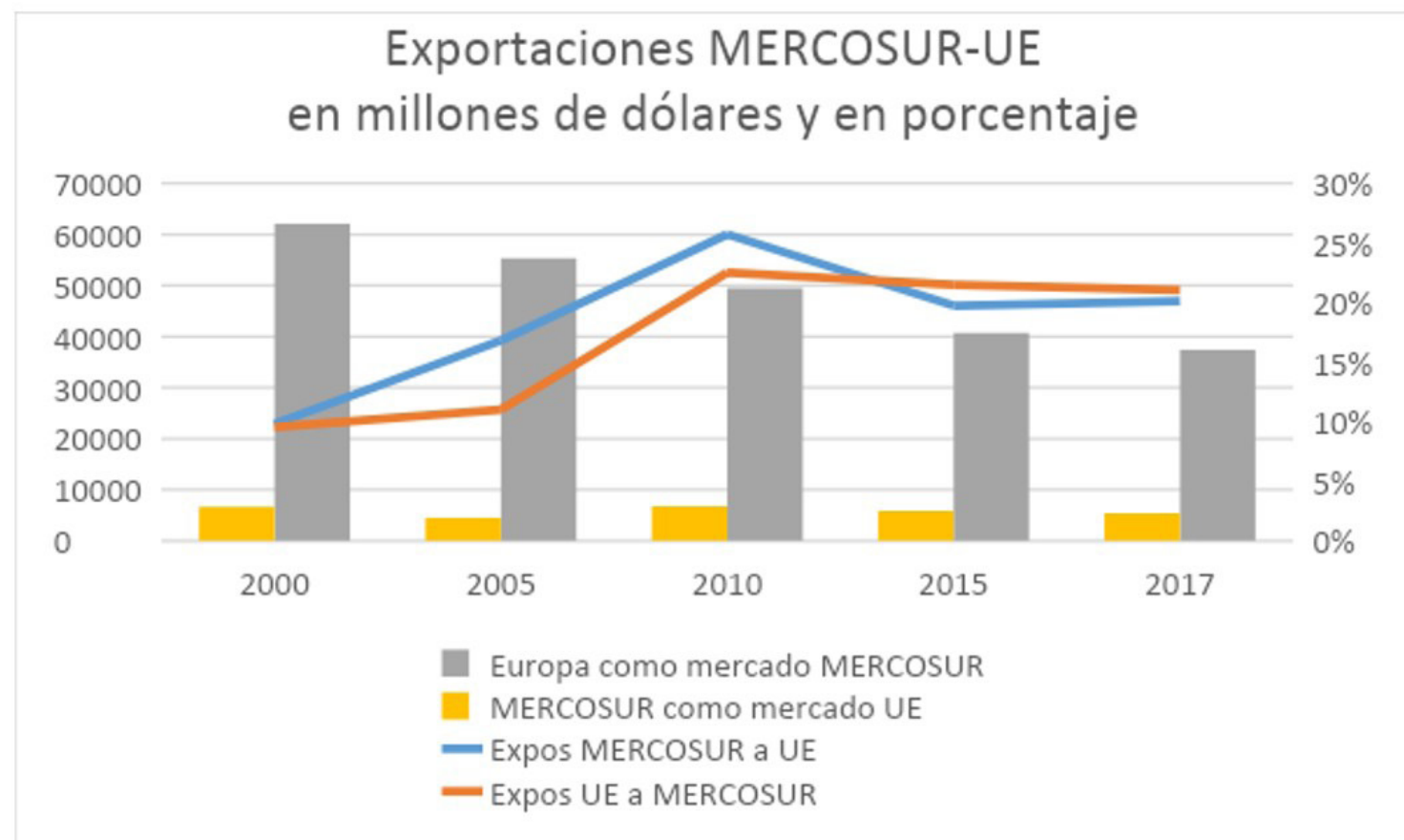

Fuente: Elaboración propia con base en datos de UN Comtrade Database

Esta complementariedad en los flujos comerciales encuentra un freno, no obstante, en las preferencias político-económicas de ambos bloques:para los países del MERCOSUR sus industrias de manufacturas son un sector sensible y protegido, a la vez que una inserción internacional "primarizante" ha resultado ser resistida por los sectores que impulsan un modelo de desarrollo

6 Adicionalmente, la UE es el principal inversor en MERCOSUR, con un stock de activos de 422,6 mil millones de dólares estadounidenses para el año 2017, según los datos de la Comisión Europea de 2019. Las inversiones de MERCOSUR en la UE eran de 57,6 mil millones de dólares estadounidenses en el mismo año.

7 En los últimos años se produjo una diversificación en los destinos de exportación del MERCOSUR. En el comienzo de la década de los 2000 los envíos a Europa llegaron a significar el $24 \%$ de las exportaciones de bienes del MERCOSUR. 
de tipo heterodoxo (Gorenstein y Ortíz, 2017). Por su parte, para la UE, el sector agrícola es un sector tradicionalmente protegido, y constituye no sólo la política común más exitosa sino también uno de los símbolos más prominentes del proyecto de integración europeo (Milward, 2000).

El promedio de los aranceles aplicados es mucho más bajo en Europa $(5,7 \%)$ que en MERCOSUR (I I,9\%). No obstante, la UE tiene los mayores picos arancelarios -de hasta $242,5 \%$ para productos lácteos- en tanto que el arancel máximo en MERCOSUR es 32,5\% -siendo el sector automotriz el más protegido (LSE Consulting, 20I8) -. Cabe señalar que si bien el MERCOSUR constituye una unión aduanera, el arancel externo común no es homogéneo y de allí que la elaboración de las listas para la negociación tenga una complejidad adicional.

Estos picos y crestas arancelarias a la vez que generaron un atractivo para impulsar las negociaciones, provocaron que el juego político detrás de tales protecciones volviera muy complejo el proceso de alcanzar un acuerdo. Ello llevó a que las negociaciones estuvieran condicionadas por las pujas sectoriales. Los sectores más activos fueron los proteccionistas, a ambos lados, siendo que desde el comienzo del proceso "los perdedores estaban organizados de forma más concentrada y asertiva que lo ganadores [del acuerdo]” (Doctor, 2007, p. 287).

Asimismo, los diferentes sectores buscaron respaldar posiciones en conjunto con sus contrapartes transatlánticas. Por ejemplo, de manera conjunta, tanto la Confederación Europea de Sindicatos como la Coordinadora de Centrales Sindicales del Cono Sur presentaron reclamos en cuanto a los contenidos sociales del acuerdo y el tratamiento de las pequeñas y medianas empresas (pymes), rechazando parte de los avances del proceso de negociación e imputándole la decisión de concluir el acuerdo al gobierno de turno (CES-CCSCS, 2018). Los agricultores europeos, como los agrupados en COPA-COGECA, se manifestaron en contra, señalando cuestiones de sustentabilidad social, económica y ambiental (COPA-COGECA, 2019). El Consejo Industrial del MERCOSUR apoyó el acuerdo siempre que éste incluyera el trato especial y diferenciado y una "cláusula de desarrollo industrial" (Consejo Industrial del MERCOSUR, 2018). Por su parte, los textiles tuvieron un desempeño excepcional al acompañar con una negociación entre privados los avances del gobierno, que luego fue utilizado como marco del acuerdo MERCOSUR-UE.

De allí que la negociación tuviese un alto grado de politización, y que los significados asociados al acuerdo desde esta perspectiva de análisis hayan estado vinculados a las disputas por los modelos de desarrollo. De hecho, existen análisis en la literatura que indican cómo las preferencias políticas se modificaron a la par que los países fueron redefiniendo sus modelos de desarrollo y preferencias institucionales (Doctor, 2007). A la hora de comprender la firma del acuerdo es necesario resaltar que mientras que las características estructurales del comercio e inversiones no variaron, ni lo hicieron las preferencias de los sectores en torno a las políticas comerciales, sí se produjo un cambio en la dimensión política de estos elementos (Sanahuja y Rodríguez, 2019). Entendemos que el hecho de que los cambios de gobiernos en los países del MERCOSUR en el período 2016-2019 hayan llevado a la adopción de modelos de desarrollo más liberales resulta un componente clave para alcanzar el cierre del acuerdo. La convergencia en el centro de la toma de decisiones de sectores con una valoración particularmente positiva de los efectos distributivos del acuerdo posibilitó el resultado que con gobiernos más neodesarrollistas 
no había resultado posible ${ }^{8}$.

El acuerdo incluye capítulos de comercio de bienes, normas de origen, defensa comercial, facilitación del comercio, obstáculos técnicos al comercio, medidas sanitarias y fitosanitarias, comercio de servicios, compras gubernamentales, propiedad intelectual, competencia, empresas del estado, subsidios, comercio y desarrollo sostenible, pymes y transparencia.Además, se incluyen una cláusula de integración regional, y una cláusula antifraude, un capítulo en materia de solución de diferencias, otro relativo a la balanza de pagos y movimiento de capital, y un anexo para el sector vitivinícola.

En materia de acceso a mercados establece que MERCOSUR eliminará sus aranceles sobre el $91 \%$ de las importaciones europeas, en tanto que la UE lo hará en un $95 \%$ - lo que ubica a las ofertas dentro de lo que había sido la expectativa inicial del bloque europeo-. En cuanto a la distribución de esos porcentajes, del lado europeo, mientras que en el sector industrial la cobertura es del 99\%, en el agrícola ésta llega al 82\%, pautándose cuotas en carnes, pollo, cerdo, azúcar, etanol, arroz, miel, maíz, queso, y leche -los sectores más sensibles y que se resistían al acuerdo-. El MERCOSUR, por su parte, ha negociado cronogramas de desgravación más prolongados para sectores como vehículos de pasajeros y calzados, pero que -a diferencia de las cuotas- en un plazo de 15 años se integrarán completamente al libre comercio entre ambas regiones. Los vinos y el sector automotor tendrán un tratamiento especial, al igual que los textiles, para los que se aplicarán normas de origen especiales (Zelicovich, 2019).

El trato especial y diferenciado inicialmente reclamado por el MERCOSUR quedó diluido en cronogramas de desgravaciones más largos, pero con un nivel de compromisos equivalente -o incluso más ambicioso dado el punto de partida - que el europeo. Europa logró preservar medidas cuantitativas sobre el comercio agrícola, donde se resalta la cuota de carne como una de las más lejanas a las ambiciones del MERCOSUR. También logró Europa que MERCOSUR alcanzase una oferta del $90 \%$, aunque el sector automotriz quedó en una canasta de desgravación de quince años de duración. La UE logró acceso a compras gubernamentales y MERCOSUR puso un umbral de acceso de 130.000 Derechos Especiales de Giro luego de un período de transición de quince años (Ministerio de Relaciones Exteriores y Culto de la Rep. Argentina, 2019a). Los negociadores del MERCOSUR lograron excluir mayores compromisos en materia de patentes dentro del capítulo de propiedad intelectual ${ }^{9}$, así como la incorporación de una novedosa cláusula de reconocimiento de indicaciones geográficas ${ }^{10}$. Las inversiones, por su parte, quedaron finalmente fuera del acuerdo (Bas Vilizzio, 2019).

Los estudios de impacto indican efectos positivos, aunque más moderados de los

\footnotetext{
8 En particular en Argentina el movimiento pendular entre modelos de desarrollo acarreó cambios en las posiciones relativas a los acuerdos de libre comercio, alternando entre estrategias más cerradas y con privilegio en las relaciones sur-sur donde el acuerdo MERCOSUR-UE perdía relevancia y sufría mayores condicionalidades; $y$ estrategias más aperturistas y favorecedoras de un enfoque norte-sur, donde el acuerdo ganaba centralidad (Actis, Lorenzini y Zelicovich, 2017)

9 En materia de propiedad intelectual, el acuerdo no incluye compromisos en extensión de patentes y protección de datos de prueba pero sí alude a que las partes hagan esfuerzos para adherir al Acuerdo de Cooperación en Patentes.

10 El acuerdo otorga nuevas protecciones a productos con indicaciones geográficas, pero incluye una suerte de "principio del abuelo" en el que productos que ya se comercializan con nombres protegidos - y que derivan en muchos casos de continuidades culturales que implicó la migración europea en Sudamérica- quedarán exceptuados.
} 
inicialmente esperados" ${ }^{\prime \prime}$ dando cuenta de cómo la duración del proceso negociador y los cambios en el sistema internacional incidieron en los potenciales efectos económicos y comerciales. El reporte de 2019 de la London School of Economics establece que un incremento del 0,1\% del PBI para la Unión Europea y un impacto mayor para el MERCOSUR, con un impacto que oscila entre el $0,1 \%$ del PBI para Paraguay en un escenario conservador hasta 0,5\% PBI para Argentina en un escenario ambicioso (London School of Economics, 2019).

El tratado entre MERCOSUR y la UE se configura entonces como un instrumento de apertura de mercados y de integración comercial entre ambas partes, siendo la variable material -económica-comercial - un componente relevante en el resultado. Del lado europeo los discursos que siguieron al $\mathbf{2 8}$ de junio enfatizaron las oportunidades generadas por este acuerdo para trabajadores y empresarios, gracias a un acceso más seguro a los mercados como también por el ahorro de cuatro mil millones de euros en pago de aranceles (Comisión Europea, 2019). En el MERCOSUR, se sostuvo que:

"La Asociación Estratégica entre Mercosur y la Unión Europea implica la integración de un mercado de $\mathbf{8 0 0}$ millones de habitantes, casi una cuarta parte del PBI mundial y con más de 100.000 millones de dólares estadounidenses de comercio bilateral de bienes y servicios" (Ministerio de Relaciones Exteriores y Culto de la Rep.Argentina, 2019b).

Sobre ello, se destacaba la existencia de oportunidades para las exportaciones, para la mejora de la competitividad —el MERCOSUR - y para la atracción de inversiones (Ministerio de Relaciones Exteriores y Culto de la Rep.Argentina, 2019a). Para Uruguay, por ejemplo, se trata de:

"un instrumento amplio, que mejora las condiciones de acceso a los mercados en el comercio de bienes y servicios, así como asegura el abastecimiento más eficiente por parte del Estado mediante el capítulo de contrataciones públicas" (Ministerio de Relaciones Exteriores de la Rep. Oriental del Uruguay, 2019).

En esta línea, la ratificación se vuelve el paso imprescindible para concretar el acuerdo. Es de esperar que las mismas pujas que acompañaron el proceso de negociaciones lo hagan ahora en el proceso de consideración por parte de los poderes legislativos, dando lugar a ratificaciones altamente conflictivas. De hecho, en el semestre siguiente a la firma tanto en Francia como en Irlanda se produjeron manifestaciones políticas en contra del acuerdo, en tanto que el Parlamento austríaco se manifestó opuesto a la ratificación del acuerdo, y el presidente francés Emmanuel Macron declaró en prensa que su gobierno no estaba listo para avanzar con la firma. Del lado del MERCOSUR el acuerdo fue un tema relevante en el proceso electoral presidencial argentino, con reverberaciones en el resto de los países miembros.

\section{El acuerdo como herramienta geopolítica}

Desde el comienzo del proceso los factores geopolíticos y estratégicos jugaron un rol importante en las negociaciones entre MERCOSUR y la UE. En el momento de lanzarse las negociaciones, las estrategias de ambas partes parecían estar sincronizadas en torno a las visiones liberales del regionalismo abierto y la globalización, con una matriz de negociación basada en el modelo de

\footnotetext{
' Años antes, el impacto estimado era mayor. Según el estudio de impacto de la Universidad de Manchester del año 2008, los beneficios económicos estáticos se estimaban en 0,5\% del PIB en Argentina, I,5\% en Brasil, 2, I\% en Uruguay, y I0\% del PIB en Paraguay.
} 
acuerdo comercial OMC-plus (Sanahuja y Rodriguez, 2019). Además, al apoyar a los esquemas subregionales de integración, la UE también promovía su estrategia interregionalista, en su proyección geopolítica. Por su parte, en términos de interacciones, en la década de los noventa, estas negociaciones estuvieron vinculadas al avance de la propuesta estadounidense del Área de Libre Comercio de las Américas (Bouzas, 2004).

Posteriormente, los factores geopolíticos y estratégicos volvieron a manifestarse cuando la evolución de la negociación se asoció a las de la Ronda Doha, plasmando esta vinculación en la noción del "bolsillo único"'2. En años más recientes, la mayor presencia de China en la región latinoamericana no puede ser descartada como un aliciente para retomar las negociaciones entre ambos bloques (Makuc, Duhalde y Rozenberg, 20I5), aunque —conforme muestra el análisis documental y de discursos - ha sido la dinámica de la crisis de globalización un factor central desde las lecturas estratégicas y geopolíticas.

La crisis de globalización - proceso en el que emergen movimientos de descontento con los pilares de la globalización y del orden liberal internacional- ha tenido un efecto notorio en el comercio. En este contexto se produjo una menor elasticidad del crecimiento de comercio en relación al crecimiento del $\mathrm{PBI}$ a la vez que avanzaron medidas proteccionistas y la inclusión de preocupaciones no comerciales en los procesos de negociación, junto a la erosión de los foros multilaterales. En este marco, los acuerdos como el de MERCOSUR-UE incrementan su valor, al volverse no solo instrumentos para preservar mayores grados de certidumbre en contextos de alza de riesgos de inestabilidad sistémica, sino que son también piezas para disputar la (re) constitución de la gobernanza global del comercio mundial.

En el contexto señalado supra, la UE se encontraba inmersa en un uso estratégico de las negociaciones comerciales internacionales, denominado "regionalismo estratégico del siglo XXl" (Zelicovich, 2018), conforme al cual había realizado ajustes sobre su estrategia que llevaron a concebir a las negociaciones como medios para fines políticos. Esta concepción se plasmó en el "Documento de reflexión sobre el encauzamiento de la Globalización" en el que la UE identificó una "oportunidad de configurar la globalización de acuerdo con nuestros valores e intereses" (Comisión Europea, 2017, p. 23) y apeló a los tratados comerciales como uno de los instrumentos para lograrlo. Se trata de una estrategia para utilizar los acuerdos comerciales como instrumento para incrementar su influencia y liderazgo global, buscando convertirse en un actor central en la preservación del libre comercio.

Las negociaciones birregionales recibieron renovado impulso en la nueva estrategia europea, y tras los cambios de gobierno en Sudamérica ${ }^{13}$, que llevaron a una nueva etapa en la agenda externa del MERCOSUR, donde MERCOSUR-UE fue una prioridad. Entre 2016 y junio

\footnotetext{
12 Este principio de single-pocket o bolsillo único impulsado por la UE buscaba limitar que la superposición de frentes negociadores erosionara su política agrícola. Sostenía que las concesiones que hiciera en el plano multilateral en las negociaciones de Ronda Doha repercutirían en el regional. Una mayor oferta en las negociaciones de Doha implicaba una menor capacidad de ofrecer concesiones frente al MERCOSUR. EI bloque mercosureño rechazaba esta vinculación (Zelicovich, 20I5).

13 Si bien es cierto que las negociaciones fueron relanzadas en 2010 bajo los gobiernos de Fernández de Kirchner y de Rouseff, ese relanzamiento no recibió el mismo énfasis político que durante los gobiernos de Macri y Temer, y primaron las condicionalidades (Makuc, Duhalde y Rozemberg, 2015). Dicha etapa había sido calificada por Peña como "una combinación de bluff game (hacer como que se negocia) con blame game (culpar a ciertos actores por la falta de avances) en la que no se está teniendo en cuenta la dimensión política estratégica” (Gaya, 20I5, P.I).
} 
de 2019 se realizaron trece reuniones del Comité Birregional de Negociaciones, coincidiendo la última con la reunión de líderes del G-20 de Osaka, Japón. Creemos que esta superposición de eventos fue clave para el desenlace del acuerdo.

La agenda de Osaka tuvo como telón de fondo a la "guerra comercial" entre Trump y Xi jinping y un "nuevo round" en la disputa por la gobernanza global. En ese marco, la meta para Donald Tusk, entonces presidente del Consejo Europeo, era posicionar a Europa como líder del multilateralismo y de un orden de comercio basado en normas (Consejo Europeo, 2019). Esta posición contrastaba con la de los otros líderes mencionados, que impulsaban metas más egoístas. De allí que el acuerdo MERCOSUR-UE fue visto -especialmente por Europa- como una pieza clave, y su uso estratégico no hubiera resultado igual de alcanzarse diez días después.

En un renovado contexto de crisis, acentuado por la disputa entre EEUU y China en los albores de la cumbre del G-20, las negociaciones se convierten en una carta por la disputa en la gobernanza global. Tal valoración puede rastrearse en los discursos de los líderes europeos de manera explícita. Por ejemplo, en ocasión del anuncio del acuerdo, el entonces presidente de la Comisión Europea, Jean-Claude Juncker, sostuvo que "en medio de las tensiones comerciales internacionales, hoy enviamos una fuerte señal junto a nuestros socios del MERCOSUR de que defendemos el comercio basado en normas" (Comisión Europea, 20 19, p. I). Por su parte, Cecilia Malmström, la entonces Comisaria Europea de Comercio, enmarcó la negociación dentro de la estrategia global, afirmando que "en los últimos años, la UE ha consolidado su posición como líder mundial en comercio abierto y sostenible. [...] Este acuerdo agrega cuatro países más a nuestra impresionante lista de aliados comerciales" (Comisión Europea, 20 I9, p. I). Más en evidencia queda esta percepción en el documento de análisis del acuerdo publicado por el Parlamento Europeo. Allí se sostiene que:

"para fines de 2018, se habían logrado pocos avances con respecto a los principales intereses ofensivos de la UE: acceso al mercado de automóviles y piezas de automóviles, productos lácteos, servicios marítimos y protección de Indicaciones Geográficas. Sin embargo, los serios desafíos planteados al sistema multilateral de comercio por el aumento del unilateralismo y del proteccionismo bajo la estrategia de America First aplicada por la Administración de EEUU desde 2017 , las incertidumbres del impacto de la guerra comercial entre EEUU y China sobre el comercio mundial, la inminente partida del Reino Unido de la UE, así como la dinámica electoral tanto en la UE como en los países del Mercosur en 2019 , se abrió una ventana de oportunidad que las partes aprovecharon el 28 de junio de 2019 para llegar a un acuerdo" (Grieger, 2019, p. 8).

Adicionalmente, para la UE, el acuerdo con MERCOSUR era una herramienta para la geopolítica regional en América Latina. En efecto, el acuerdo puede leerse asimismo como una manera de contrabalancear la influencia de EEUU en América Latina y enviar un mensaje a la administración Trump - reacia a entablar negociaciones comerciales con socios latinoamericanos(Saraiva, 20I2, p.54), y a su bloqueo a los acuerdos mega-regionales (TPP y TTIP). En esta misma línea, puede considerarse como una respuesta a la creciente influencia de China en la región (Ortiz Velázquez y Dussel Peters, 2016). EI MERCOSUR también parecía estar interesado en recuperar espacios en el ámbito internacional de la mano del principal bloque comercial del mundo, en una 
especie de estrategia de bandwagoning.

Como hemos señalado, una de las particularidades de la dimensión estratégica y geopolítica como driver de un acuerdo comercial es su volatilidad. Así, cuando tras la Cumbre de Osaka el impacto se diluyó y la crisis de globalización entró en un breve lapso de mayor tranquilidad por un posible acuerdo entre EEUU y China, la fuerza de estas variables como impulsoras del acuerdo disminuyó. De hecho, luego de la Cumbre de Osaka, las tensiones subyacentes de la variable material volvieron a emerger, generando rumores vinculados a una no ratificación del acuerdo en cuestión, tanto por sensibilidades agrícolas como ambientales —del lado europeo- y en menor medida, de trabajadores e industriales, en el MERCOSUR. El acuerdo como herramienta geopolítica cumple sus objetivos con el mero anuncio del acuerdo en principio en la coyuntura particular analizada, pero para su ratificación vuelve a quedar preso de la politización inherente a la variable material.

\section{El relato identitario del acuerdo MERCOSUR-UE}

Al analizar la variable identitaria en las relaciones MERCOSUR-UE debemos diferenciar tres cuestiones. Primero, la cuestión de la identidad del MERCOSUR, especialmente en su dimensión externa; segundo, la proyección externa de la identidad de la UE y las consecuencias para su estrategia regionalista e interregionalista. Finalmente, la posibilidad de crear identidades compartidas entre estos dos actores a través del interregionalismo ${ }^{14}$.

La dimensión externa de la identidad del MERCOSUR, tiene como eje la agenda externa de negociaciones con terceros países. Los estados partes deben dedicar tiempo y recursos al ejercicio de identificación de intereses comunes, el establecimiento interno de prioridades y la defensa externa de las posiciones acordadas (Oelsner, 20I3).De manera intermitente, el dinamismo de la agenda exterior del bloque ha compensado la falta de progresos en el plano interno. Así, mientras que a nivel interno el propósito de la asociación se estaba diluyendo, los estados partes encontraron una fuente de cohesión en torno a las negociaciones externas (Oelsner, 20I3). En este sentido, las conversaciones interregionales con la UE constituyeron uno de los procesos externos más importantes del bloque en términos de formación de identidad (Oelsner, 2013) ${ }^{15}$ y actuaron como un "pegamento" indispensable en favor de la coherencia y unidad del bloque (Bianculli, 2020).

Por su parte, la proyección internacional de la UE se vincula con la singularidad del carácter de "potencia normativa" de la UE (Manners, 2002) — que destaca el proyecto cosmopolita de gobernanza promovido a partir de su propia experiencia de integración-y que basa en los valores de democracia, justicia social, imperio de la ley,y un diálogo igualitario con las contrapartes regionales en sus iniciativas interregionales (Sanahuja, 2007, pp. 9-10).

En los últimos tiempos, la credibilidad y la legitimidad de la UE como "potencia normativa" se han erosionado por diferentes factores. Lejos de haber completado una unión, la UE está

\footnotetext{
14 Esta idea de "convergencia de valores" o "identidades compartidas" rara vez se somete a investigaciones empíricas: se trata de un argumento conceptual, derivado de juicios abstractos dictados sobre la base de una retórica interregionalista recurrente.

15 Poco después de haber firmado el acuerdo con la UE, el bloque sudamericano pudo cerrar las negociaciones para un acuerdo comercial con la Asociación Europea de Libre Comercio.
} 
lidiando con varias crisis desde hace algunos años, en ámbitos como la economía, las finanzas, el empleo, las migraciones y la seguridad, la pérdida de un estado miembro y enfrentando fuertes cuestionamientos a su legitimidad política (Álvarez, 2017).

El infortunado manejo que ha hecho Bruselas de las sucesivas crisis que golpean al bloque erosiona su política normativa en el exterior y su estrategia de relación interregional con arreglo a valores. Otro factor que ha debilitado el modelo europeo y su capacidad de influencia para reivindicar en el exterior una política orientada al mercado, pero a la vez, apegada a ciertos valores y principios, es la existencia de un “doble estándar". Por ejemplo, las políticas de promoción de la democracia, el desarrollo y la cohesión social con criterios básicos de derechos humanos, por un lado, y los acuerdos de libre comercio, con sus estrictas reglas de protección de inversiones o de derechos de propiedad intelectual, por otro, constituyen un ejemplo de la actuación interregional de la UE en sus relaciones con América Latina. La UE pasó a ser percibida en nuestra región como un actor "neoliberal" no muy diferente a EEUU (Sanahuja, 20I2).

Si bien, como indicamos anteriormente, la importancia de un sentido común de pertenencia estructura, al menos en lo retórico, las relaciones de la UE con el MERCOSUR, con la firma del acuerdo de 2019 el bloque europeo demostró otorgar prioridad a la agenda comercial. La economía volvió a ser el vector impulsor de la acción exterior de la UE, lo que significa mayor atención a intereses económicos y empresariales, por encima de consideraciones políticas como la democracia y los derechos humanos (Sanahuja, 20I2).

Los valores, así como las identidades, son dinámicos y cambiantes, y específicos según el contexto y el momento (Wunderlich, 20I2). La fórmula discursiva recurrente de convergencia de valores como la defensa de los derechos humanos y la democracia, parece reorientarse a la promoción del multilateralismo eficaz y del libre comercio. El informe antes mencionado del Parlamento Europeo destaca que:

"En el contexto de una vuelta a las relaciones de poder en el comercio, el unilateralismo y el proteccionismo, la Unión Europea ha pretendido enviar una señal inequívoca al mundo de que ambas partes apoyan el sistema comercial multilateral basado en normas y refuerzan la cooperación estratégica [...]" (Grieger, 2019, p. 7).

La tercera cuestión está estrechamente vinculada con la anterior. Como sabemos, la tradición discursiva del interregionalismo entre la UE y el MERCOSUR insiste en que la esencia de estos vínculos son los valores comunes que ya forman parte del acervo común (Gratius, 20 I0). En plenas conversaciones en 2017, la UE consideraba que con el MERCOSUR "[...] pensamos en el mismo sentido y que con quien más comparte la Unión Europea sus valores fundamentales es con los países del Mercosur y de América Latina" (Rivas Molina, 2017, p.2). Sin embargo, hay una cuestión metodológica fundamental que no se percibe en la mayor parte de la literatura: los valores e identidades comunes constituyen el punto de partida, y no de llegada, del interregionalismo.

Por estas razones, la variable identitaria en el vínculo interregional MERCOSUR-UE alcanzaría para fundamentar el mantenimiento de las negociaciones por dos décadas a pesar de las dificultades de las mismas, pero no logra explicar la finalización de las negociaciones comerciales, 
y tampoco la (posible) ratificación del acuerdo. La tesis identitaria como elemento explicativo del resultado del acuerdo de 2019 pierde sustento dado que la coincidencia de valores y principios es un supuesto subyacente siempre presente en estas relaciones y no un "resultado" de las mismas. Los países europeos y los del MERCOSUR disfrutan de un legado cultural e histórico que los une de antemano, y eso es justamente lo que diferencia estos lazos interregionales de cualquier otro tipo.

El factor de identidad en la explicación del comportamiento cooperativo interregional constituye, en este sentido, una condición previa para la cooperación (Hveem, 2003). Los valores, principios e ideas que informaron la decisión de establecer una asociación estratégica en los años noventa explican el mantenimiento del proceso de negociaciones (pese a las interrupciones) —dado que el enfoque del MERCOSUR en su interregionalismo con la UE fue "esencialmente emulativo y reactivo a las estructuras y pretensiones globales de la UE”' (Gardini, Koschut y Falke, 2018, p. 2I3) - pero no logran dar cuenta de la conclusión de las negociaciones comerciales.

\section{Conclusiones}

El acuerdo MERCOSUR-UE no admite una única lectura, sino que —como hemos analizadopuede explicarse por la presencia de al menos tres factores explicativos. Cada uno de éstos tiene además sus propios efectos sobre los usos estratégicos del mismo, y permite inferir su proyección sobre el proceso de ratificación según se presentan como estructurales o coyunturales a los actores. No pueden entenderse los resultados de junio de 2019 desde lo económico, lo geopolítico o lo identitario exclusivamente. Haber puesto en diálogo estas tres variables ha resultado indispensable para aprehender la complejidad de un proceso de larga data, y las implicancias del mismo en la eventual ratificación.

En el comienzo de las negociaciones tanto los factores económico-comerciales como los geopolíticos e identitarios se conjugaron para hacer posible y explicar el lanzamiento de un ambicioso proyecto de asociación birregional. Como se desarrolló en el trabajo, el elemento identitario logra explicar el sostenimiento de la negociación en el tiempo -incluyendo en ello el hecho de que tras las suspensiones se volvieran a retomar los intercambios de ofertas-. Los intereses económicos y comerciales tienen un rol más ambiguo: dan cuenta tanto del impulso como del bloqueo de las negociaciones. Por su parte, observamos una correlación entre el debilitamiento del factor geopolítico y los períodos de impasse de las negociaciones. Finalmente, para entender el cierre del acuerdo, es este factor —el geopolítico- el que vuelve a emerger con fuerza en los últimos meses, junto a los factores identitarios y el realineamiento de las fuerzas en pugna en la variable material hacia la orientación más liberal, en ambos lados del Atlántico.

Cada uno de estos factores ha implicado además un "uso" del acuerdo comercial para las partes involucradas, sugiriendo un distinto grado de conflictividad en el siguiente proceso de ratificación. Del lado económico-comercial hemos constatado cómo el acuerdo ha sido presentado como un instrumento para el acceso a los mercados; como una oportunidad para incrementar el bienestar y eficiencia económica de los distintos sectores económicos. La concreción de este relato requiere de la ratificación del acuerdo, pero es, por las sensibilidades involucradas, la que acarrea un mayor nivel de politización para ese proceso.

La geopolítica también ha jugado un rol relevante, especialmente en el uso estratégico del 
anuncio del acuerdo en el marco de la Cumbre de Osaka.Así, ha sido presentado como un hito para la conservación del orden liberal internacional, en contraste con la dinámica de la guerra comercial sino-americana. Este juego estratégico, sin embargo, queda satisfecho con el mero anuncio de la asociación estratégica y la variable geopolítica no genera los mismos incentivos para la ratificación como lo hizo para la culminación de las negociaciones.

Por último, la explicación basada en el elemento identitario se construye a partir del interregionalismo permite sostener que el acuerdo entre MERCOSUR y la UE ha sido leído como la consolidación de una identidad común en torno a un conjunto de valores compartidos. Pero, como hemos señalado, éstos son una precondición para que el acuerdo se lleve a cabo, y no explican en su totalidad el resultado de junio de 2019. Esta interpretación de los actores no genera necesariamente incentivos hacia la ratificación, aunque genera precondiciones para facilitarla. El acuerdo, ratificado o no, es un elemento central para la historia del vínculo interregional y para la proyección internacional de las partes involucradas. No obstante, ello dependerá, en gran parte, de la interacción de los tres factores aquí estudiados ${ }^{16}$.

El trabajo pone de manifiesto, así, que en un contexto donde las negociaciones comerciales internacionales son cada vez más complejas y donde en sí misma la variable comercial ha dejado de ser un tema de low agenda (Harding y Harding, 2020), un análisis multicausal con marcos conceptuales eclécticos presenta ventajas para la comprensión de casos como el del cierre de negociaciones entre MERCOSUR y UE. Con este abordaje, la investigación realizada ha permitido echar luz sobre las tensiones subyacentes al momento de la firma del acuerdo, y que comienzan a manifestarse en el proceso de ratificación. Muestra también que sobre un mismo hecho de política internacional -el acuerdo comercial- coexisten distintas miradas y motivaciones por parte de los actores involucrados.

En esta línea, una de las posibles perspectivas para enriquecer el estudio del acuerdo MERCOSUR-UE, y en particular profundizar la comprensión sobre el proceso de cierre de las negociaciones, es su análisis desde los estudios de politización (Mckibben y Taylos, 20I4; Zürn, 2019; Meunier y Roederer-Rynning, 2020). El trabajo de Bianculli (2020) genera un antecedente relevante para esta línea, habiendo la autora indagado en el rol de la politización al momento del relanzamiento de las negociaciones en 2010. También, dado que nuestra investigación alcanza a sugerir una vinculación entre factores explicativos y usos del acuerdo comercial, se desprende la posibilidad de avanzar en una línea de estudios comparados de otras negociaciones comerciales que permitan ahondar en los alcances y límites de esta vinculación.

\section{Referencias}

Actis, E., Lorenzini, M. E.,y Zelicovich, J. (2017). La vinculación entre modelo de desarrollo y estrategia de inserción en la Argentina democrática (1983-20II), Studia Politicae, 4I, pp. I05-I 35.

Álvarez, M.V. (20|3). La crisis de la Unión Europea analizada desde una perspectiva neofuncionalista: lecciones para la integración regional en América Latina, Perspectivas Internacionales, 9 (2), pp. I28-163.

Álvarez, M.V. (2017). ¿Cuál es el rumbo futuro de la Unión Europea? El debate en torno a los diferentes caminos posibles, Cuadernos de Política Exterior Argentina (Nueva Época), 126, pp. 87-94.

\footnotetext{
${ }_{16} \mathrm{Al}$ momento de concluir este trabajo el acuerdo MERCOSUR-UE se encontraba en el proceso de revisión legal, restando su firma y posterior
} envío a los respectivos parlamentos. 
Bas Vilizzio, M. (2019). Acuerdo Mercosur-Unión Europea: sombras y ausencia de la solución de controversias inversor-Estado. Documento de Trabajo 21 ( $2^{a}$ época), Madrid: Fundación Carolina. Recuperado de: https:// fundacioncarolina.es/wp-content/uploads/20I9/II/DT_FC_21.pdf

Bianculli, A. (2020). Politicization and Regional Integration in Latin America: Implications for EU-MERCOSUR Negotiations?, Politics and Governance, 8 (I), PP. 254-265

Bouzas, R. (2004). Las negociaciones Unión Europea-MERCOSUR. Entre la lentitud y la indefinición, Nueva Sociedad 190, pp. I25-135

Bouzas, R. y Zelicovich, J. (20I4). La Organización Mundial de Comercio, los acuerdos mega-regionales y los usos estratégicos del regionalismo, Estudios de Economía Aplicada, 32 (3), pp. 963-994.

CES-CCSCS. (febrero 2018). Carta a los negociadores del acuerdo UE-Mercosur. Recuperado de: https://www.cta.org.ar/ IMG/pdf/declaracion_ces_y_ccscs_.pdf (I2.II.20I9)

Consejo Industrial del Mercosur. Negociaciones MERCOSUR - Unión Europea. (20I8). Recuperado de: https:// uiaorgar-cmsdev.s3.amazonaws.com/pdf/Declaracion_CIM_Asuncion.pdf?X-Amz-Algorithm=AWS4HMAC-SHA256\&X-Amz-SignedHeaders=host\&X-Amz-Credential=AKIAJXL23AJMKLGIZFNA\%2F20I9I2 04\%2Fus-east- I\%2Fs3\%2Faws4_request\&X-Amz-Date=2019|204TI $901 \mathrm{I} \mid \mathrm{Z} \& X-A m z-E x p i r e s=$

Comisión Europea (2019). EU and Mercosur reach agreement on trade. Press Release. Bruselas, 27 de junio. Recuperado de: https://ec.europa.eu/commission/presscorner/detail/en/IP_I9_3396

Comité de Negociaciones Birregionales MERCOSUR - Unión Europea (2000).Acta de la primera reunión del Comité de Negociaciones Birregionales MERCOSUR - Unión Europea. Buenos Aires, 6 y 7 de abril. Recuperado de: http://www.sice.oas.org/TPD/MER_EU/negotiations/CNB_I_s.pdf

Consejo Europeo (2019). G20 summit in Osaka, Japan, 28-29 de junio. Recuperado de: https://www.consilium.europa. eu/en/meetings/international-summit/2019/06/28-29/

COPA-COGECA (2019). EU-Mercosur - Exiting Commission opens Pandora's box of double standards in agriculture. CONFAGRI. Recuperado de: https://www.confagri.pt/content/uploads/2019/07/EU-mercosur-exitingcommission-opens-Pandoras-box-of-double-standards.._.pdf

Dieter, H. (20I4). The Return of Geopolitics:Trade Policy in the Era of TTIP and TPP. Berlin: Friedrich-Ebert-Stiftung, Global Policy and Development.

Doctor, M. (2007).Why Bother with Inter-Regionalism? Negotiations for a European Union-MERCOSUR Agreement, Journal of Common Market Studies, 45 (2), pp. 28I-3I4.

Farrell, M., Hettne, B. y Van Langenhove, L. (2005), Global politics of regionalism. Theory and Practice, Londres: Pluto Press.

Gaya, R. (20I5). Presentación de la Nota Técnica "La negociación MERCOSUR-Unión Europea a veinte años del acuerdo marco de cooperación: Quo vadis?”. Conexión Intal, columna de análisis n²29. Recuperado de: https://conexionintal.iadb.org/2015//0//5/presentacion-de-la-nota-tecnica-la-negociacion-mercosur-unioneuropea-a-veinte-anos-del-acuerdo-marco-de-cooperacion-quo-vadis-2/

Gilpin, R. (200I). Global Political Economy. Understanding the international economic order. New Jersey: Princeton University Press.

Gómez-Mera, L. (2009). Domestic constraints on regional cooperation: Explaining trade conflict in MERCOSUR, Review of International Political Economy, 16 (5), pp. 746-777.

Gorenstein, S. y Ortiz, R. (2017). El nuevo ciclo de primarización en el Cono Sur latinoamericano. Aportes para una aproximación crítica, Revista Interdisciplinaria de Estudios Agrarios, 46, Pp. 14I-160. Recuperado de: http://I57.92.I36.59/download/riea/riea_v46_nl_05.pdf

Gratius, S. (2010). Las agendas gubernamentales de las cumbres Unión Europea y América Latina y el Caribe: funcionalidad y cohesión limitadas, en Cienfuegos, M.y Sanahuja,J.A. (eds.) Una región en construcción. UNASUR y la integración en América del Sur, Barcelona: Fundación CIDOB, pp. 389-404.

Grieger, G. (2019). The trade pillar of the EU-Mercosur Association Agreement. Bruselas: European Parliamentary Research Service. Recuperado de: https://www.europarl.europa.eu/RegData/etudes/BRIE/2019/640 I38/ EPRS BRI(2019)640I38 EN.pdf

Gruber, L. (200I). Power politics and the free trade bandwagon, Comparative Political Studies, 34 (7), pp. 703-74I.

Hänggi, H., Roloff, R. y Rüland, J. (2006). Interregionalism and International Relations, Londres y Nueva York: Routledge Taylor \& Francis Group.

Harding, R., y Harding, J. (2020). Strategic Trade as a Means to Global Influence. en Klasen, A., The Handbook of Global Trade Policy. Oxford: John Wiley \& Sons, pp. I43-I72.

Hettne, B. (20l4). Regional Actorship: A Comparative Approach to Interregionalism, en Baert, F., Scaramagli, T. y Söderbaum, F. (eds.), Intersecting Interregionalism. Regions, Global Governance and the EU. Dordrecht: Springer, pp. $55-70$.

Hveem, H. (2003). The Regional Project in Global Governance, en Söderbaum, F. y Shaw, T.M. (eds.), Theories of New Regionalism. A Palgrave Reader, Basingstoke: Palgrave Macmillan, pp. 8I-98.

Laïdi, Z. (2008). How Trade Became Geopolitics, World Policy Journal, 25 (2), pp- 55-6I.

Laursen, F. (2008). Theory and practice of regional integration, Jean Monnet/Robert Schuman Paper Series, 8 (3), pp. I-22. Recuperado de: http://aei.pitt.edu/8219///LaursenLongSympos08RegIntegedi.pdf

London School of Economics (2019). Sustainability Impact Assessment in support of the Association Agreement Negotiations Between the European Union and MERCOSUR. Draft Interim Report. Londres: LSE. Recuperado de: http://www. eumercosursia.com/uploads/4/0/7/2/40728425/final_interim_report_publication_03oct2019.pdf 
LSE Consulting (2018). Sustainability Impact Assessment in support of association agreement negotiations between the European Union and Mercosur. Inception Report. Londres: LSE Consulting. Recuperado de: https://trade. ec.europa.eu/doclib/docs/20I8/march/tradoc_I5663I.pdf

Makuc, A., Duhalde, G., y Rozenberg, R. (20I5). La Negociación MERCOSUR-Unión Europea a veinte años del Acuerdo Marco de Cooperación: Quo Vadis?. Buenos Aires: BID-Intal.

Malamud,A. (2018). Overlapping Regionalism, No Integration: Conceptual Issues and The Latin American Experiences, Politica Internacional, 3 (6), pp. 46-59

Manners, I. (2002). Normative Power Europe: A Contradiction in Terms?, Journal of Common Market Studies, 40, pp. 235-258.

Mansfield, E., y Milner, H. ( I999). The new wave of regionalism, International Organization, 53 (3), pp. 589-627.

Mattli, W. (1999). The Logic of Regional Integration: Europe and Beyond. Cambridge: Cambridge University Press. doi: $10.1017 / C B O 9780511756238$

Mckibben, H. y Taylos, T. (agosto, 20I4). Let's Talk About Trade:The Politicization and Framing of International Trade Policy. Ponencia preparada para "American Political Science Association Conference",Washington.

Meissner, K. (20I5). ¿Ha fracaso el interregionalismo? Las negociaciones UE-ASEAN, Revista CIDOB d’Afers Internacionals, II0, pp |4-4|.

Meunier, S. y Roederen-Rynning, C. (2020). Missing in Action? France and the Politicization of Trade and Investment Agreements, Politics and Governance, 8 (I), pp. 3I2-324. DOI: I0.17645/pag.v8i I.26I6

Milner, H. (1999). The Political Economy of International Trade, Annual Review of Political Science, 2, pp. 9 I - I I 4.

Milward, A. S. (2000). The European rescue of the Nation-State, Segunda edición, Londres y Nueva York: Routledge, [I a edición de 1994]

Ministerio de Relaciones Exteriores y Culto de la República Argentina (2019a). Acuerdo de Asociación MERCOSUR - Unión Europea. Resumen de contenidos del pilar comercial. Recuperado de: https://www.cancilleria.gob.ar/ acuerdo-mercosur-ue/resumen-de-contenidos-del-pilar-comercial

Ministerio de Relaciones Exteriores y Culto de la República Argentina (2019b). Mercosur cierra un histórico Acuerdo de Asociación Estratégica con la Unión Europea. 28 de junio. Recuperado de: https://www.cancilleria.gob.ar/ es/actualidad/noticias/mercosur-cierra-un-historico-acuerdo-de-asociacion-estrategica-con-la-union

Ministerio de Relaciones Exteriores de la República Oriental del Uruguay (2019).Acuerdo de Asociación MERCOSURUnión Europea - Síntesis del acuerdo. Julio Recuperado de: https:/www.gub.uy/ministerio-relacionesexteriores/sites/ministerio-relaciones-exteriores/files/inline-files/SINTESIS\%20DEL\%20ACUERDO\%20 DE\%20ASOCIACION\%20MERCOSUR\%20Y\%20UNION\%20EUROOPEA.pdf

Moravcsik, A. (1998). The choice for Europe. Social purpose and state power from Messina to Maastricht. Abingdon: Routledge.

Oelsner, A. (2013). The Institutional Identity of Regional Organizations, Or Mercosur's Identity Crisis, International Studies Quarterly 57, pp. II5-127

Organización Mundial de Comercio (20II). Informe sobre Mundial 20II La OMC y los acuerdos comerciales preferenciales: de la coexistencia a la coherencia. Ginebra:WTO press.

Ortiz Velásquez, S. y Dussel Peters, E. (20I6). La nueva relación comercial entre América Latina y el Caribe y China: ¿promueve la integración o desintegración comercial? en Dussel Peters, E. (coord.), La nueva relación comercial entre América Latina y el Caribe-China: ¿integración o desintegración regional? México: Unión de Universidades de América Latina y el Caribe, pp. I3-58.

Rivas Molina, F. (23 de marzo de 2017). La Unión Europea y Mercosur avanzan en los 'valores fundamentales' de un acuerdo, El País. Recuperado de: https://elpais.com/economia/2017/03/23/actualidad/I490296947_327534. html

Rüland, J. (20I4). International relations and Interregionalism: Reanimating an Obsolescent Research Agenda?, en Baert, F., Scaramagli, T. y Söderbaum, F., (eds.) Intersecting Interregionalism. Regions, Global Governance and the EU. Dordrecht:, Springer, pp. I5-35.

Sanahuja, J.A. (2007). Regiones en construcción, interregionalismo en revisión. La UE y el apoyo al regionalismo y la integración latinoamericana, en Freres, C., Gratius, S., Mallo, T., Pellicer, A. y Sanahuja, J. A. (eds.): ¿Sirve el diálogo político entre la Unión Europea y América Latina?, Documento de Trabajo, I5, Madrid: Fundación Carolina, pp. I-42.

Sanahuja,J.A. (20I2). Las cuatro crisis de la Unión Europea, Anuario Ceipaz 2012 - 20 I 3. Cambio de ciclo: crisis, resistencias y respuestas globales, Madrid: CEIPAZ, pp. 5 I-84.

Sanahuja, J.A. y Rodríguez, J. D. (2019). Veinte años de negociaciones Unión Europea - MERCOSUR: Del interregionalismo a la crisis de la globalización, Documento de Trabajo I3, Madrid: Fundación Carolina.

Saraiva, M. G. (20I2). Brazil's Strategies and Partnerships: The Place of the European Union, Perspectives 20 (2), pp. 45-62.

Söderbaum, F. y Van Langenhove, L. (2005). Introduction:The EU as a Global Actor and the Role of Interregionalism, Journal of European Integration, 27 (3), pp. 249-262.

Wunderlich, J. (20I2). The EU an Actor Sui Generis? A Comparison of EU and ASEAN Actorness, Journal of Common Market Studies, 50 (4), pp. 653-669.

Zelicovich, J. (20I5). Juntos, pero no tanto: un recorrido por la agenda de negociaciones comerciales externa del MERCOSUR (I99|-20I5), Revista Integración y Cooperación Internacional, 2I, pp. 16-26. 
Zelicovich, J. (2018). Usos estratégicos de las negociaciones comerciales internacionales en un orden de hegemonías en disputa, Brazilian Journal of International Relations, 7 (3), pp. 692-7I 7.

Zelicovich,J.(2019). El Acuerdo MERCOSUR-Unión Europea en su recta final. Madrid: Fundación Carolina. Recuperado de: https://www.fundacioncarolina.es/wp-content/uploads/2019/07/AC-I3.pdf

Zürn, M. (2019). Politicization compared: at national, European, and global levels, Journal of European Public Policy, 26 (7), pp. 977-995. 


\section{RELACIONES INTERNACIONALES}

Revista académica cuatrimestral de publicación electrónica Grupo de Estudios de Relaciones Internacionales (GERI)

Universidad Autónoma de Madrid, España

https://revistas.uam.es/relacionesinternacionales

ISSN 1699 - 3950

f facebook.com/RelacionesInternacionales

3. twitter.com/RRInternacional

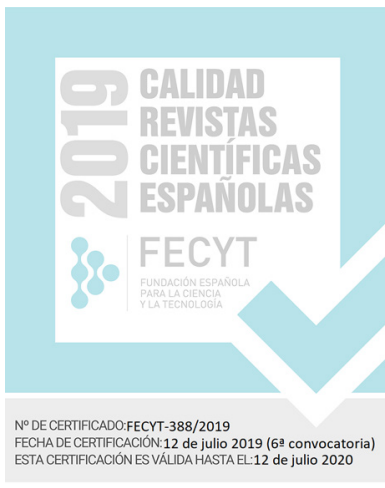

